\title{
Removal of OH Spectral Interferences from Aqueous Solvents in Inductively Coupled Plasma-Atomic Emission Spectrometry (ICP-AES) with Ar Cryogenic Desolvation
}

\author{
Youngmin Cho and Yong Nam Pak* \\ Department of Chemistry, Korean National University of Education, Chung-Buk363-791, Korea.*E-mail: pakyn@knue.ac.kr \\ Received May 26, 2005
}

\begin{abstract}
The spectral interferences of $\mathrm{OH}$ from aqueous solvents in ICP-AES have been studied and eliminated using a cryogenic argon trap. The prominent lines of Bi I $306.772 \mathrm{~nm}$, Al I $309.271 \mathrm{~nm}$, and V II $310.230 \mathrm{~nm}$, which are very seriously overlapped with the $\mathrm{OH}$ band, were examined. With an extended torch and high tangential flow of $20 \mathrm{~L} / \mathrm{min}$, water vapor from air entrainment was prevented. The combination of a condenser and argon cryogenic trap was able to eliminated most of water vapor carried by the argon sample gas. Removal of $\mathrm{OH}$ spectral interference could extend the linearity of the calibration curve 5-10 times on the lower concentration for ICP-AES. Interference Equivalent Concentration (IEC) has been reduced to 5.6, 5.9, and 12.4 times for Bi, $\mathrm{Al}$ and $\mathrm{V}$, respectively.
\end{abstract}

Key Words : Cryogenic desolvation, Spectral interference, Solvent removal, ICP-AES

\section{Introduction}

Removal of water vapor could bring many advantages in Inductively Coupled Plasma Spectroscopy such as an increased Signal-to-Noise ratio $(\mathrm{S} / \mathrm{N}))^{1,2}$ The noise level in the background was reduced because of the removal of large droplets that can travel high up in the plasma causing large fluctuations. ${ }^{1}$ Another aspect of water removal that has been most widely utilized is to reduce solvent loading. ${ }^{3-10}$ Since a common pneumatic nebulizer uses only $1 \%$ of liquid sample, sensitivity could be increased by simply introducing more aerosols into plasma with a high efficiency nebulizer such as an ultrasonic nebulizer (USN). ${ }^{3,4}$ Water loading was more significant in an axial viewed ICP-AES. ${ }^{7}$

A removing or substantial reducing of water loading is mandatory if a commonly used low power of $1 \mathrm{~kW} \mathrm{ICP} \mathrm{is} \mathrm{to}$ be used. Several researches have used different desolvation systems such as a cooled spray chamber, ${ }^{11}$ a heater and condenser $1,{ }^{12}$ membrane dryer, ${ }^{13-16}$ cryogenic desolvation ${ }^{17-20}$ as well as the combination of two to improve the analytical performance of ICP. Especially in the use of organic solvents, to remove solvent loading is required for a low power ICP and beneficial even for a high power ICP. Wiedrin $^{21}$ and others ${ }^{22}$ utilized cryogenic desolvation to improve the detection capability of ICP in the use of organic solvents while Gustavsson ${ }^{23}$ used a membrane interface.

Another important advantage of removing solvent vapor is that it can reduce or remove spectral backgrounds from molecular species associated with a solvent. In this paper, removal of $\mathrm{OH}$ interference is described. Water as well as acid is pyrolized in the plasma and make various polyatomic species such as $\mathrm{OH}, \mathrm{NO}$, and $\mathrm{NH}$ to interfere with analytical lines or peaks. ${ }^{24,25}$ In ICP-Mass Spectrometry (MS), molecular species such as oxide, hydride and hydroxide can seriously overlap with analytical peaks. ${ }^{26}$ Chlorinated molecules ${ }^{27}$ could be a serious problem for a hydrochloric acid containing solvent as well. It has been demonstrated in
$\mathrm{ICP}_{\mathrm{MSS}}{ }^{27,28}$ that a cryogenic desolvation could be very useful in removing these backgrounds and precise measurement of several elements. To get rid of all spectral interferences due to the water solvent, both aerosols and water vapor must be removed. In addition to a condenser, a cryogenic desolvation is required to lower the solvent vapor pressure because even water vapor at the room temperature can cause interference to a large degree. However, utilizing this technique on ICP-AES has not been easily found in the literatures.

Several molecules and radicals give serious spectral interferences on the analytically useful wavelength range. Oxygen shows strong absorbance below $200 \mathrm{~nm}$, which is not a primary concern for most studies. However, NH emission occurs around $336 \mathrm{~nm}$ and NO shows intense bands from 200 to $280 \mathrm{~nm}$, with the 237.0, 247.9, and 272.2 $\mathrm{nm}$ bands head being the strongest. $\mathrm{OH}$ has a wide range of rotational vibrational spectrum ranging from 281.0 to 294.5 $\mathrm{nm}$ and from 306.0 to $324.5 \mathrm{~nm}$. There are many important elements that are interfered in these spectral ranges as well.

A high-resolution spectrometer can alleviate the problem to some degree. Boumans ${ }^{22}$ examined Al I $309.271 \mathrm{~nm}$ that was interfered with $\mathrm{OH} 309.278 \mathrm{~nm}$ and a high resolution showed more than 3.5 times improvement in detection limits than a medium resolution spectrometer. For a concentration that gives more than $10 \%$ error, it improved more than 4.5 times. However, it would be impossible to correct if the interfering line is directly overlapped with the analytical line.

Since $\mathrm{OH}$ has the broadest range, it has been chosen as a primary target of this study. Bi I $306.772 \mathrm{~nm}$, Al I 309.271 $\mathrm{nm}$, and V II $310.230 \mathrm{~nm}$ are some of the examples that are seriously overlapped with $\mathrm{OH}$ rotational spectrum. They are chosen for this study because they are either the strongest or the second strongest lines in the list, thus, easy to demonstrate the effect of cryogenic condenser. However, there are more than 20 other elements, of which intensities are strong 
or medium, that are interfered rather strongly in this spectral range. Though their intensities may not be the strongest, the interference could be more severe and the improvement is more obvious.

Usually when a selected wavelength is interfered strongly, a second line could be chosen for the analysis conveniently. However, this technique can offer an alternative choice of analytical line, when the alternative line chosen is not strong or even has large interferences. Furthermore, it could be extended to other spectral interferences caused by water solvent such as NH and NO. Especially when the solvent is organic, cryogenic cooling would be even more useful in removing the interferences from $\mathrm{C}(\mathrm{I}), \mathrm{CN}, \mathrm{CO}$, and $\mathrm{C}_{2}$.

The primary object of this research is to demonstrate the capability of removing $\mathrm{OH}$ spectral interferences to extend the analytical capability of ICP-AES.

\section{Experimental Section}

Instrumentation. A Perkin Elmer model Plasma 1000 ICP system (Perkin-Elmer, CT, USA) was used for the experiment. The monochromator was equipped with a grating 2400 grooves $/ \mathrm{mm}$ and its linear dispersion was 0.38 $\mathrm{nm} / \mathrm{mm}$. The photomultiplier tube was Hamamatsu R787. To prevent air moisture from coming into the plasma, an extended torch $3 \mathrm{~cm}$ taller than a normal torch was used. Tangential gas flow rate was increased to $20 \mathrm{~L} / \mathrm{min}$ to reduce air diffusion into the plasma. Carrier gas flow rate, sample uptake rate, and auxiliary gas flow rate were the same as the

Table 1. Experimental apparatus and optimal operating conditions used in the experiment

\begin{tabular}{ccl}
\hline $\begin{array}{c}\text { Conditions of spectrometer } \\
\text { Monochromator }\end{array}$ & & \\
Focal length & $:$ & $1 \mathrm{~m}$ \\
Grating & $:$ & 2400 grooves $/ \mathrm{mm}$ \\
Spectral range & $:$ & $160-775 \mathrm{~nm}$ \\
Linear dispersion & $:$ & $0.38 \mathrm{~nm} / \mathrm{mm}$ \\
Grating size & $:$ & $84 \times 84 \mathrm{~mm}^{2}$ \\
PMT & $:$ & Hamamatsu R787 \\
Dynamic range & $:$ & $10^{6}$ \\
& & \\
Conditions of complete desolvation system \\
ICP torch & $:$ & extended torch $(3 \mathrm{~cm}$ extended $)$ \\
Observation position & $:$ & $10 \mathrm{~mm}$ above the coil \\
Power & $:$ & $1.0 \mathrm{~kW}$ \\
Argon gas flow rate & & \\
Outer & $:$ & $20 \mathrm{~L} / \mathrm{min}$ \\
Auxiliary & $:$ & $1.0 \mathrm{~L} / \mathrm{min}$ \\
Nebulizer & $:$ & $1.0 \mathrm{~L} / \mathrm{min}$ \\
Nebulizer & $:$ & \\
Sample uptake rate & $:$ & $1.0 \mathrm{~mL} / \mathrm{min}$ \\
Cryogenic trap temp. & $:$ & $-180^{\circ} \mathrm{C}$ \\
Condenser temperature & $:$ & $-20{ }^{\circ} \mathrm{C}$ \\
Heater temperature & $:$ & $180^{\circ} \mathrm{C}$ \\
\hline
\end{tabular}

normal analysis condition. Detailed operating conditions are summarized in Table 1.

The Ar cryogenic desolvator was made locally using Pyrex $^{\circledR}$. All connection tubing ( $0.95 \mathrm{~mm}$ o.d., $0.80 \mathrm{~mm}$ i.d.) was made of PTFE (Polytetrafluoroethylene). In Figure 1, the cryogenic desolvation system used in this study is shown. It consists of two heaters, a condenser, and a cryogenic argon trap. A Liebig type condenser was used with a coolant of ethylene glycol water mixture $(6: 4)$ to keep the temperature $-20{ }^{\circ} \mathrm{C}$ with a thermo bath (model RBC-10, Jeio Tech. Co. LTD, Seoul, Korea). Water was condensed as an ice and could block the sample flow. At the sample flow rate of $1.0 \mathrm{~m} / \mathrm{min}$, it could be operated for 2 hours without any significant blockage. A Pyrex ${ }^{\circledR}$ column (i.d. $9 \mathrm{~mm}$, o.d. $12 \mathrm{~mm}$ ) was placed before the condenser and heated to be $180^{\circ} \mathrm{C}$ at the exit. Heating was done with a heating tape wrapped around the column. The temperature, which was controlled by a variac, was measured by placing a regular glass thermometer at the surface of the column beneath the heating tape. After the condenser, it was necessary to have second heating applied before the cryogenic trap to minimize re-absorbing of vapor to salt particles. The trap was constructed with Pyrex ${ }^{\circledR}$ as shown in Figure 1 and was immersed in liquid Ar. Since liquid $\mathrm{N}_{2}$ has a lower boiling point (b.p. $-198.9{ }^{\circ} \mathrm{C}$ ) than Ar, it could condense Ar gas. Only Ar or He trap could be used. The total length of the aerosol path was about $1.5 \mathrm{~m}$.

Reagent. Stock solutions of 1,000 ppm were made and diluted with $1.5 \%$ nitric acid or hydrochloric acid (Dong Woo Chemicals, Seoul, Korea), which are normally used for semiconductor wafer cleansing. The levels of impurities in acids were found satisfactory for ICP-MS and this research. Bismuth and Aluminum solutions were made from $99.99 \%$

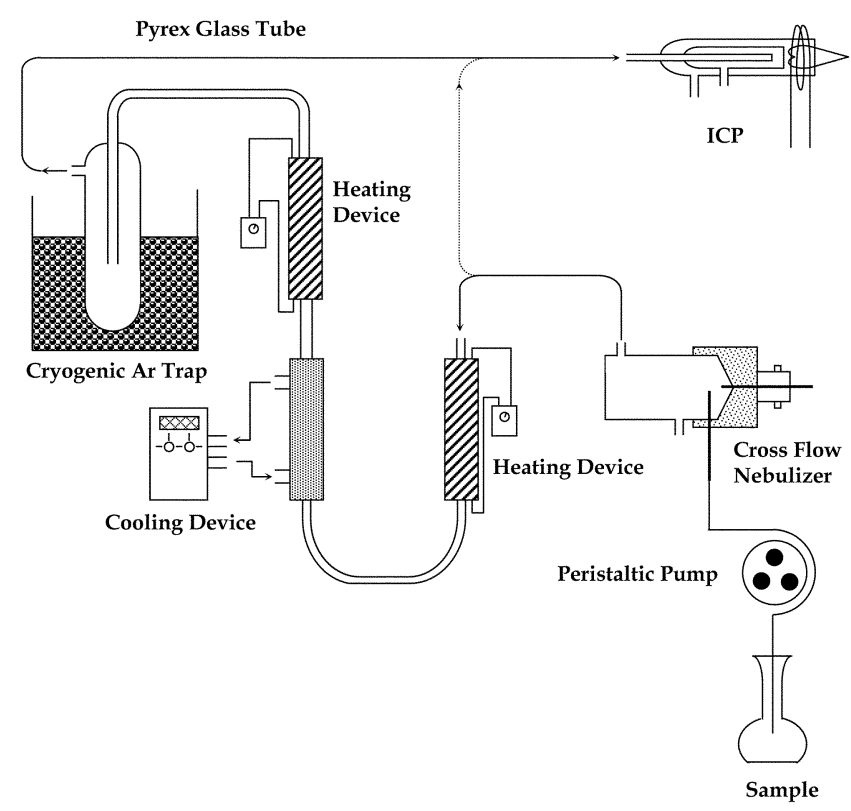

Figure 1. Desolvation system used in the experiment. The system is consisted of a condenser, two heater and temperature controllers, and Ar cryogenic trap. The total length of desolvation system is 1.5 $\mathrm{m}$ long. 
pure metals (Spex, USA) and Vanadium was from ammonium vanadate. High purity water $(18 \mathrm{M} \Omega / \mathrm{cm}$ resistivity) was obtained from Millie-Q Water System (Millipore, Bedford, MA, USA).
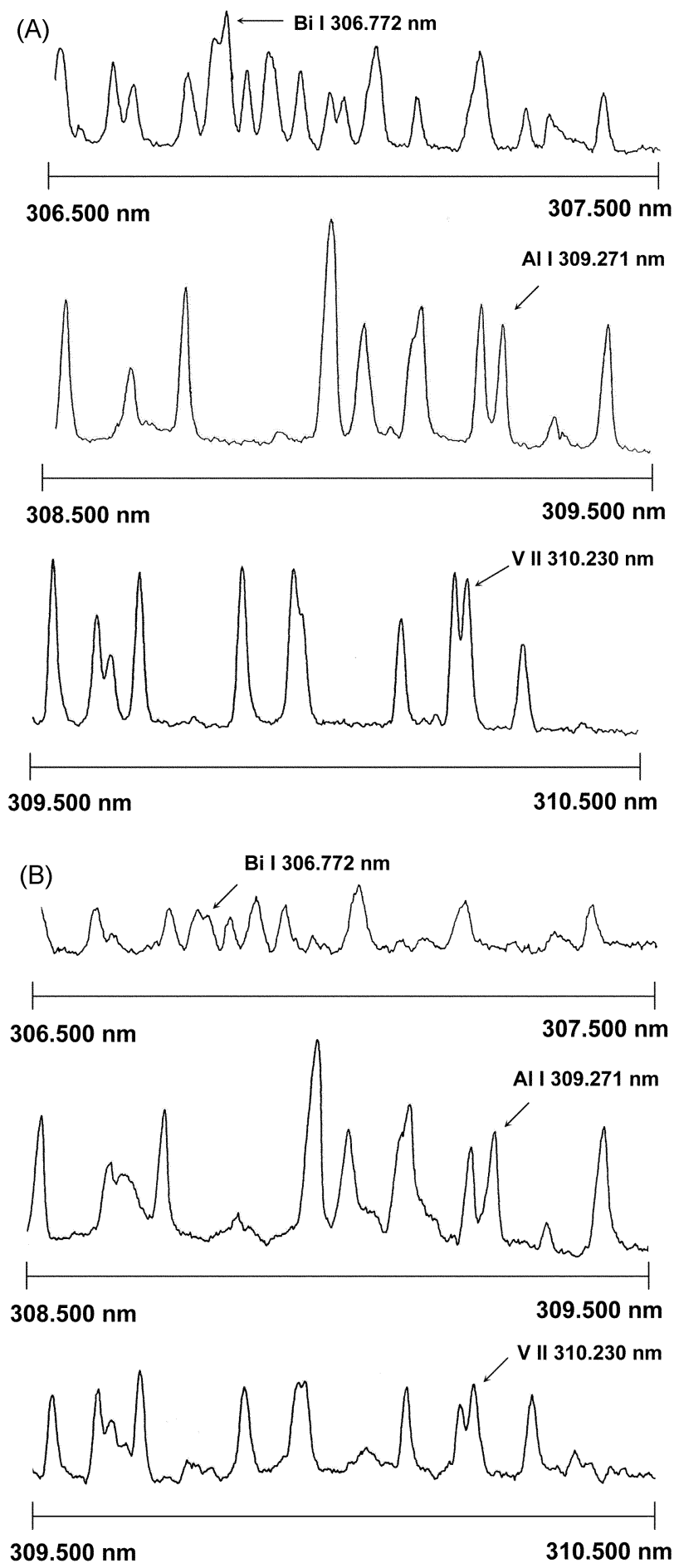

Figure 2. (a) $\mathrm{OH}$ band interferences on Bi I $306.772 \mathrm{~nm}$, Al I $309.271 \mathrm{~nm}$, V II $310.230 \mathrm{~nm}$ with aqueous solvent nebulization. Experimental conditions: Normal torch; Observation height: 15 $\mathrm{mm}$ above load coil; Outer gas flow rate: $15 \mathrm{~L} / \mathrm{min}$; Sample uptake rate: $1 \mathrm{~mL} / \mathrm{min}$. (b) $\mathrm{OH}$ band interferences on $\mathrm{Bi} \mathrm{I} 306.772 \mathrm{~nm}$, Al I $309.271 \mathrm{~nm}$, V II $310.230 \mathrm{~nm}$ without nebulization. Other experimental conditions are the same as $2-a$.

\section{Results and Discussion}

Figure 2 shows $\mathrm{OH}$ band spectrum obtained under normal analytical conditions (outer gas flow rate: $15 \mathrm{~L} / \mathrm{min}$ ) at observation height $15 \mathrm{~mm}$ above the load coil for "wet" (Fig. 2-a) and "dry" plasma (Fig. 2-b). A "wet" plasma means water is nebulized into the plasma while "dry" plasma means no solvent is nebulized. As shown in Figure 2, the $\mathrm{OH}$ lines directly overlap upon $\mathrm{Bi}, \mathrm{Al}$ and $\mathrm{V}$ wavelengths and the interference could be severe for both plasmas. The peak intensities are several times larger for a "wet" than "dry" plasma. The sensitivity scale for Figure 2-b is several times larger than Figure 2-a. It is quite surprising that even though the interferences are expected to be absent in "dry" plasma, they still exist as shown in Figure 2-b. It is suspected that they are from air moisture and impurities from Argon gas supply. No other interferences from $\mathrm{NO}, \mathrm{N}_{2}{ }^{+}$, and $\mathrm{NH}$ overlap in this region of $\mathrm{OH}$.

Effect of tangential gas and extended torch. Tangential gas flow rate was increased to reduce the water vapor entrainment from air. Figure 3 shows decrease of $\mathrm{OH}$ interferences with the change of the outer gas flow for VII at $310.230 \mathrm{~nm}$. It shows that the $\mathrm{OH}$ background was reduced significantly at $20 \mathrm{~L} / \mathrm{min}$ and changed little or became worse at $25 \mathrm{~L} / \mathrm{min}$. Probably, too much turbulent gas flow can cause air entrainment. The same pattern was observed for $\mathrm{Bi}$ and $\mathrm{Al}$. Winge ${ }^{29}$ was also able to reduce $\mathrm{OH}$ background drastically by increasing the outer gas flow rate from $10 \mathrm{~L} /$ $\min$ to $20 \mathrm{~L} / \mathrm{min}$. However, increasing tangential flow rate alone was not enough to completely remove $\mathrm{OH}$ lines even at "dry" plasma.

The extended torch was used to further reduce air entrainment in the plasma. As shown in Figure 4, the $\mathrm{OH}$ spectrum was almost diminished (compare with Fig. 2-b) and interfered very little with analytical lines. All of the experiment was performed at this condition of $20 \mathrm{~L} / \mathrm{min}$ outer gas flow rate and $10 \mathrm{~mm}$ above the load coil with the
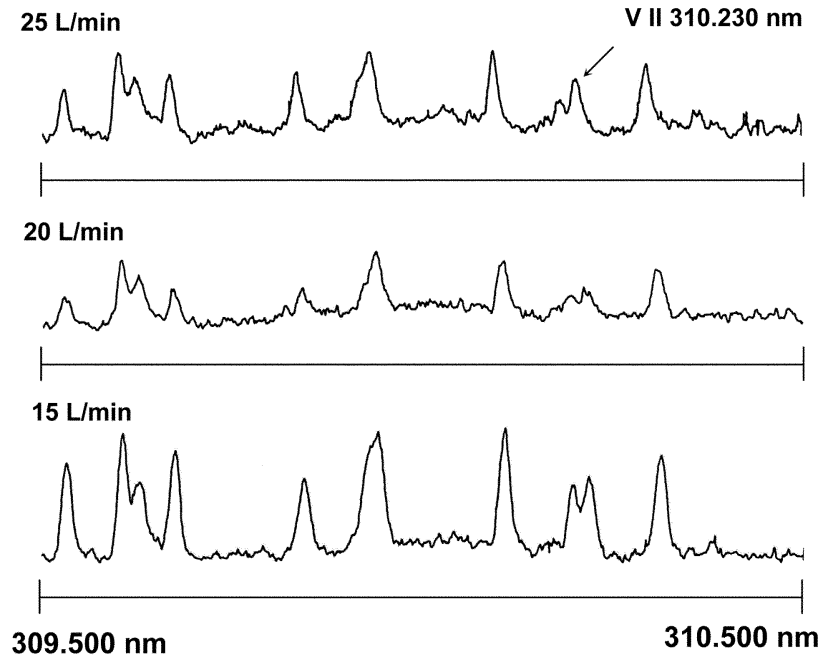

Figure 3. Depression of $\mathrm{OH}$ interference on Bi I $306.772 \mathrm{~nm}$ for three tangential gas flow rates $15 \mathrm{~L} / \mathrm{min}, 20 \mathrm{~L} / \mathrm{min}$, and $25 \mathrm{~L} / \mathrm{min}$. Experimental conditions: normal torch; plasma: dry. 


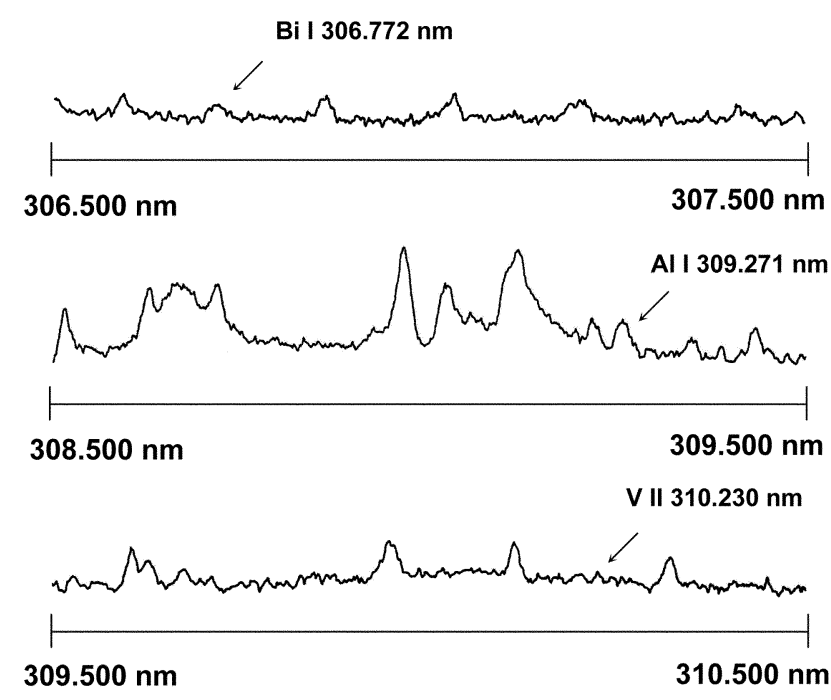

Figure 4. OH spectrum over $1.000 \mathrm{~nm}$ spectral window for Bi I $306.772 \mathrm{~nm}$, Al I $309.271 \mathrm{~nm}$, V II $310.230 \mathrm{~nm}$ for dry plasma using the extended torch. $\mathrm{OH}$ interference is completely removed with the use of high tangential gas flow rate and extended torch.

extended torch. OH spectrum from a dry argon gas, Figure 4, could be considered as a reference. If water is completely removed with the desolvation system and the vapor entrainment is prevented, the spectrum obtained should be the same as Figure 4. Observation at the lower region of plasma could reduce the background even when a normal torch is used. However, signal-to-background ratio was deteriorated significantly due to the background increase and the loss of signal from insufficient ionization.

Removal of $\mathrm{OH}$ spectrum in a "wet" plasma. First, a condenser was used only to remove water and its spectrum is shown in Figure 5. Dotted lines represent $\mathrm{OH}$ lines and show strong interferences on analytical lines. The efficiency of water removal by the condenser was determined to be $70 \%$ in this study similar to other reports. ${ }^{30,31}$ The remained 30\% water contributes as $\mathrm{OH}$ background strongly. As was shown in Figure 3 and 4, even small amount of moisture can cause a strong interference. Tough the use of a condenser can remove some water, strong $\mathrm{OH}$ lines remain due to incomplete removing of water and its vapor. Interestingly, the $\mathrm{OH}$ line intensity decreased $50 \%$ only instead of $70 \%$. This could suggest that there was water loading still remained in I kW ICP. ${ }^{9}$

Saiwa ${ }^{32}$ showed that solvent molecules can be absorbed on the surface of salt particles even after they pass through the heater and condenser. The higher the solubility of the salt, the more water the aerosol mist carried. When a combination of heater and condenser is used alone, the vaporized solvent present along with the wet salt particle. Thus, it was required to reheat the dried sample before the particles enter into the Ar cryogenic trap.

The necessity of using Ar cryogenic trap is explained like following. The cryogenic argon trap could further reduce the amount of water vapor introduced in ICP by lowering the saturation vapor pressure of the water solvent even after a

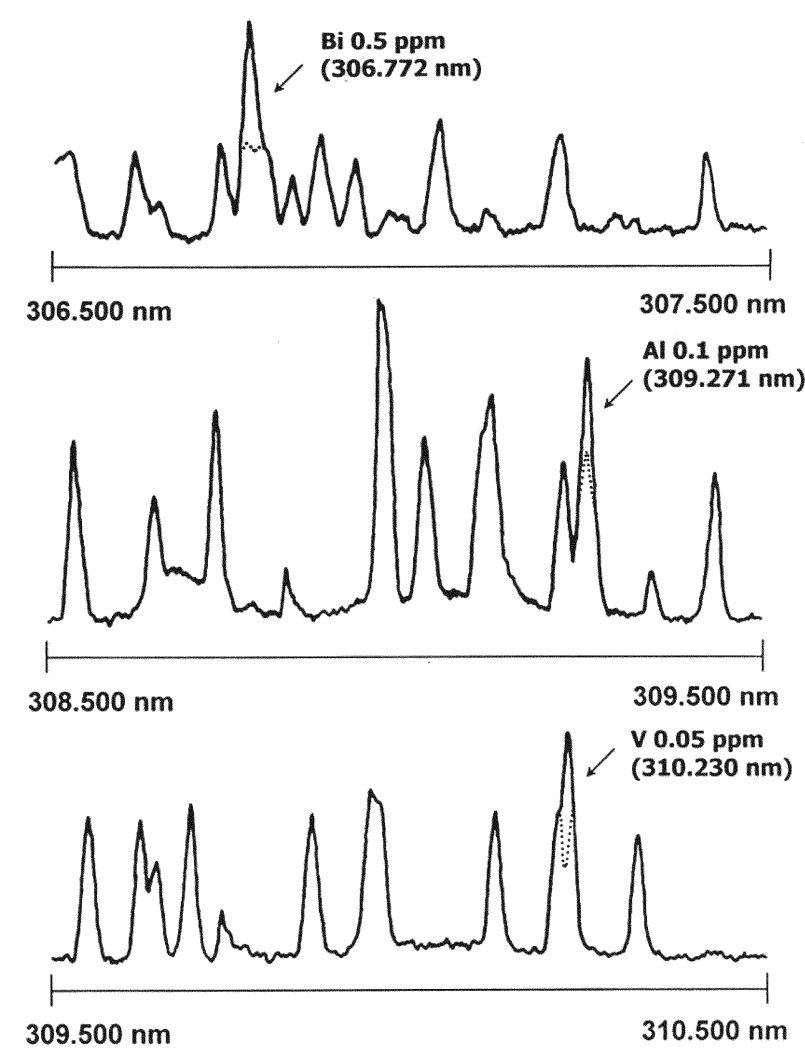

Figure 5. Signals and $\mathrm{OH}$ band interferences on $\mathrm{Bi} \mathrm{I} 306.772 \mathrm{~nm}$, Al I $309.271 \mathrm{~nm}$, V II $310.230 \mathrm{~nm}$ when a condenser is used only. The dotted lines are $\mathrm{OH}$ background interferences on analytical lines. Experimental conditions: Extended torch; Outer gas flow rate: $20 \mathrm{~L} / \mathrm{min}$.

condenser. The amount of water introduced into ICP is a sum of nebulized aerosol and vapor. When a typical nebulization efficiency of $1 \%$ is used and the sample flow rate is $1.0 \mathrm{~mL} / \mathrm{min}$, about $0.01 \mathrm{~g} / \mathrm{min}$ of aerosol is sent into ICP by a nebulizer. The amount of water carried as vapor can be calculated using the ideal gas law. The water vapor at $25{ }^{\circ} \mathrm{C}$ is calculated as $0.023 \mathrm{~g} / \mathrm{min}$ assuming $1.0 \mathrm{~L} / \mathrm{min}$ gas flow. At $0{ }^{\circ} \mathrm{C}$, it is drastically reduced to $0.00484 \mathrm{~g}$. However, the vapor still consists $30 \%$ of the total water that goes into ICP. Water condenser alone can never remove vapor complete. The vapor pressure must be lowered by a cryogenic desolvation. To make vapor less than $1 \%$ of total water amount, the temperature should be decreased below $230 \mathrm{~K}$.

Figure 6 shows $\mathrm{OH}$ spectrum obtained when both the condenser and the cryogenic trap were in operation. Outer gas flow rate was $20 \mathrm{~L} / \mathrm{min}$ and the observation height was $10 \mathrm{~mm}$ above the load coil. If the removal is complete, then the $\mathrm{OH}$ spectrum obtained with cryogenic trap will be the same as the "dry" argon gas spectrum. When compared with Figure 4, which shows the OH spectrum of "dry" plasma, background peak intensities are almost the same, which proves that the system is efficient in removing $\mathrm{OH}$ background interference.

One may argue that the sensitivity may decrease due to the use of condenser and cryogenic trap. Of course, sample is 


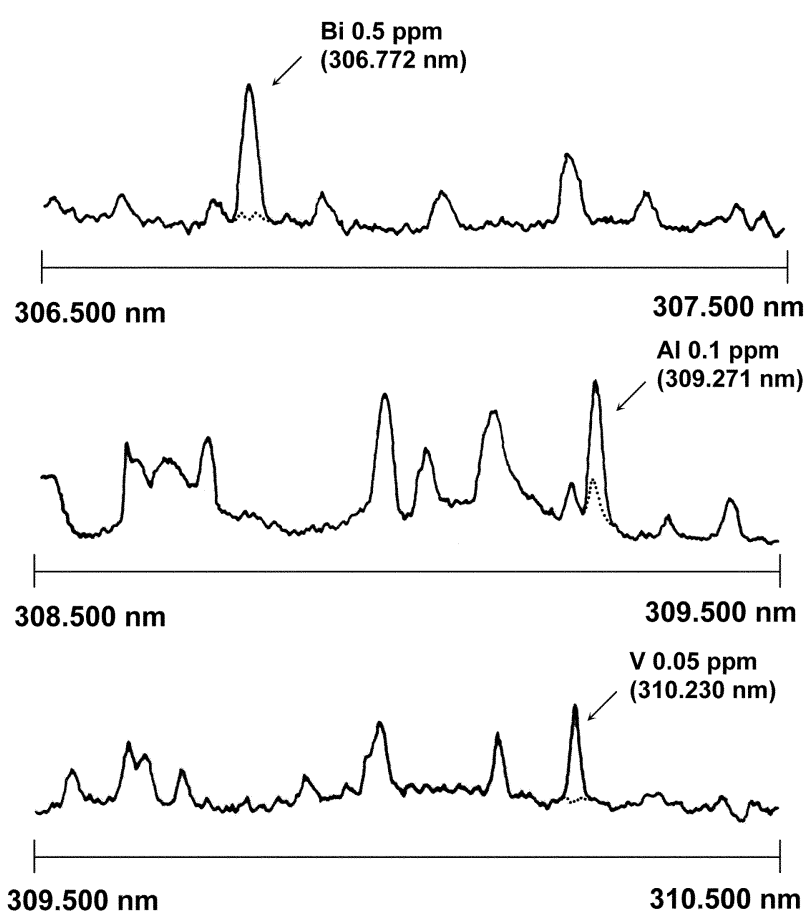

Figure 6. Removal of $\mathrm{OH}$ interference in wet plasma with $\mathrm{Ar}$ cryogenic desolvation system for Bi I $306.772 \mathrm{~nm}$, Al I 309.271 $\mathrm{nm}$, V II $310.230 \mathrm{~nm}$. The dotted lines are $\mathrm{OH}$ background. When compared with Figure 4, the Ar cryogenic trap is quite effective in removing $\mathrm{OH}$ interferences. Experimental conditions: Extended torch; Outer gas flow rate: $20 \mathrm{~L} / \mathrm{min}$.

lost in the desolvation process. The amount of analyte loss was determined by introducing $\mathrm{Bi} 50 \mathrm{ppm}$. The desolvation system (condenser and cryogenic trap) was washed with 100 $\mathrm{mL}$ of water after nebulizing $\mathrm{Bi}$ for 100 minutes. The sample loss rate calculated was $11.7 \pm 4.0 \%(n=5)$. Though it is disadvantageous to loose sample, when a cryogenic trap is used, it is better off in determining analytes of low concentration by removing $\mathrm{OH}$ interference. Introducing more sample to increase sensitivity is not the answer when there is solvent spectral interference. Only complete removing of solvent would be the solution and this type of research in ICP-AES has rarely been done.

Calibration curve and improvement of IEC (Interference Equivalent Concentration). IEC could be defined $^{21}$ as the equivalent concentration of analyte that gives the same intensity of interference. It shows the degree of interference from other lines and can be used as an index of improvement by the cryogenic system. Usually, detection limits (DLs) are used to examine the performance improvement of a system. However, in this study, removal of interference does not necessarily improve DL because it is related to increased intensity and/or decreased noise in background. Consequently, IEC has been used in this study to show the improvement by the removal of interference.

Though ICP-AES shows a good linearity of the calibration curve, it is bent easily at low concentrations due to the background. Consequently, background correction is mandatory at low concentrations. Since the interference of $\mathrm{OH}$ is
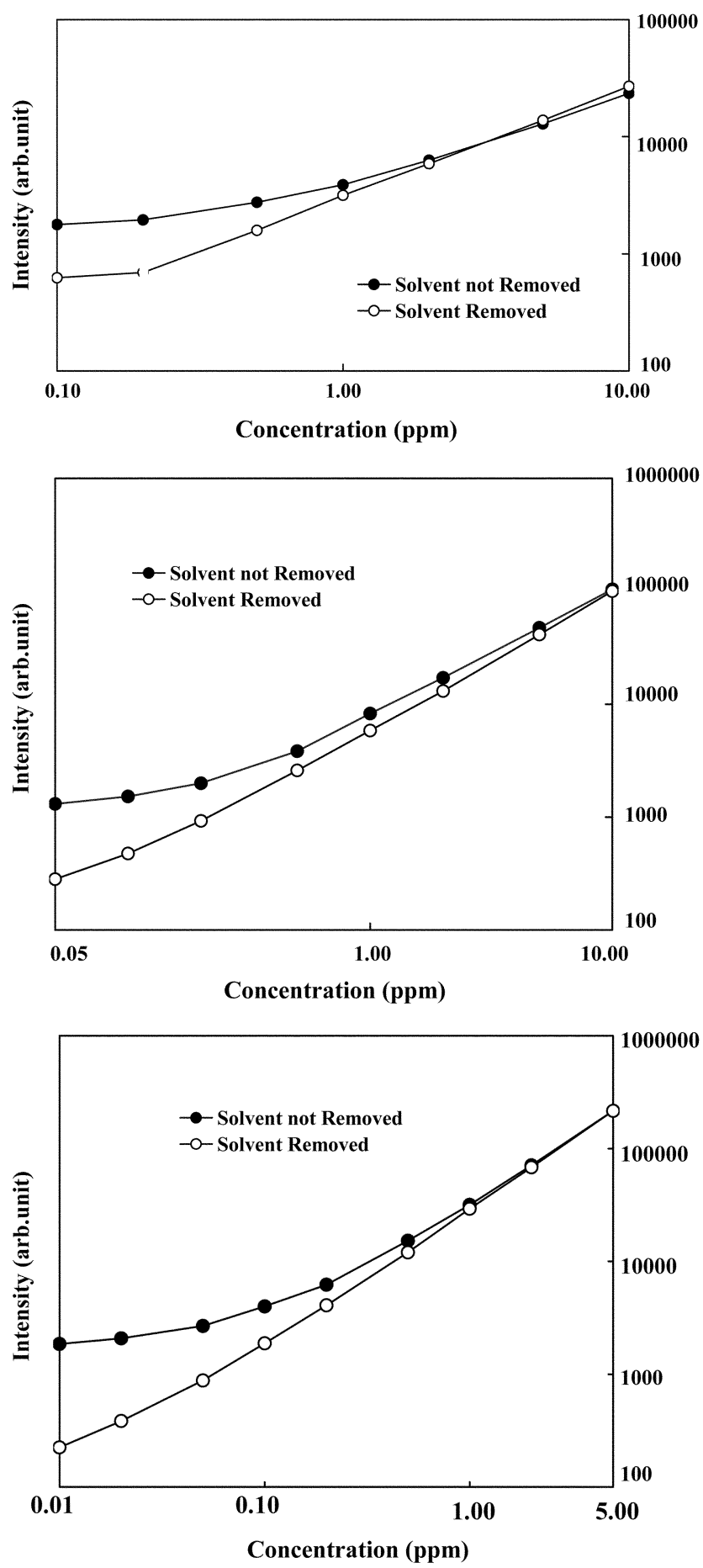

Figure 7. (a) Calibration curve of Bi I $306.772 \mathrm{~nm}$ for wet ( $\bullet$ ) and dry ( $\bigcirc$ ) plasmas. (b) Calibration curve of Al I $309.271 \mathrm{~nm}$ for wet (•) and dry ( $\bigcirc)$ plasmas. (c) Calibration curve of V II $310.230 \mathrm{~nm}$ for wet $(\bullet)$ and dry $(\bigcirc)$ plasmas.

relatively high, a correct concentration cannot be determined especially at low concentration levels. The linearity of calibration curve for the $\mathrm{OH}$-free plasma was examined and shown in Figure 7. Calibration curves of Bi I $306.772 \mathrm{~nm}$ (Fig. 7-a), Al I $309.271 \mathrm{~nm}$ (Fig. 7-b), and V II $310.230 \mathrm{~nm}$ (Fig. 7-c) with and without $\mathrm{OH}$ removal are shown. When water was removed, the linearity was extended 5 to 10 times 
Table 2. IEC (Interference Equivalent Concentration) improvement using a complete desolvation system unit: $\mathrm{mg} / \mathrm{L}$

\begin{tabular}{lcc}
\hline & Solvents not Removed & Solvents Removed \\
\hline Bi I 306.772 nm & 0.172 & 0.0307 \\
Al I 309.271 nm & 0.271 & 0.0456 \\
V II 310.230 nm & 0.0812 & 0.00657 \\
\hline
\end{tabular}

toward lower concentration.

Table 2 shows IEC for elements studied. IEC was calculated from the comparison of analytical intensity to interference intensity. Removal of water vapor brought improvement factors of 5.6, 5.9, 12.4 for $\mathrm{Bi}, \mathrm{Al}$ and $\mathrm{V}$, respectively. Since the $\mathrm{OH}$ interference line intensity was stronger for $\mathrm{V}$, removal of interference gave a better improvement factor for Vanadium.

\section{Conclusion}

The spectral interferences of $\mathrm{OH}$ in ICP-AES have limited one to compromise for weaker analytical lines or to sacrifice intensity for a higher resolution. With a combination of condenser and the cryogenic desolvation, water could be almost completely removed. An extended torch and high outer gas flow were needed to remove air entrainment and residual $\mathrm{OH}$ interferences in the plasma. From the comparison with dry plasma, it was concluded that the $\mathrm{OH}$ molecular spectrum was fully suppressed.

It was examined for those lines that seriously overlapped with $\mathrm{OH}$ rotational spectrum first. IEC was reduced for Bi I $306.772 \mathrm{~nm}$, Al I $309.271 \mathrm{~nm}$, V II $310.230 \mathrm{~nm}$ by 5.6, 5.9, 12.4 times, respectively, when the cryogenic trap was used. The linear dynamic range of $\mathrm{Bi}$ I $306.772 \mathrm{~nm}$, Al I 309.271 $\mathrm{nm}$, V II $310.230 \mathrm{~nm}$ was extended 5 to 10 times in lower concentration region. Since there are more than 20 other elements of which lines are interfered, this study should be further extended for them. Of course, this study can be applied to the removal of molecular species such as NH or $\mathrm{NO}$ as well as other spectral range of $\mathrm{OH}$. Any other solvents, especially the organic solvent, can be removed to eliminate complicating spectral interferences.

Acknowledgment. This research has been supported by NON DIRECTED RESEARCH FUND, Korea Research Foundation.

\section{References}

1. Olesik, J. W.; Smith, L. J.; Williamsen, E. J. Anal. Chem. 1989, $61,2002$.

2. Nixon, D. E. J. Anal. At. Spectrom. 1990, 5, 531 .

3. Tsukahara, R.; Kubota, M. Spectrochim. Acta 1990, 45B, 581.

4. Olson, K.; Haas, W. J.; Fassel, V. A. Anal. Chem. 1977, 49, 632.

5. Douglass, K. O.; Fitzgerald, N.; Ingebrethsen B. J.; Tyson, J. F. Spectrochim. Acta 2004, 59B, 107.

6. Huang, M.; Kojima, H.; Shirasaki, T.; Hirabayashi, A.; Koizumi, H. Anal. Chim. Acta 2000, 413, 217.

7. Masson, P.; Vives, A.; Orignac D.; Prunet, T. J. Anal. At Spectrom. 2000, 15, 543.

8. Novotny, I.; Farinas, J. C.; Wang, J.; Poussel, E.; Mermet, J.-M. Spectrochim. Acta 1996, 51B, 1517.

9. Luo, S. K.; Berndt, H. Spectrochim. Acta 1994, 49B, 485.

10. Koropchak, J. A.; Winn, D. H. Appl. Spectrosc. 1987, 41, 1311.

11. Hutton, R. C.; Eaton, A. N. J. Anal. At. Spectrom. 1987, 2, 595.

12. Castilluno, T. M.; Vela, N. P.; Caruso, J. A.; Story, W. C. J. Anal. At. Spectrom. 1992, 7, 807.

13. Tao, H.; Miyazaki, A. J. Anal. At. Spectrom. 1995, 10, 1.

14. Yang, J.; Conver, T.; Koropchak, J. A.; Leighty, D. A. J. Anal. At. Spectrom. 1996, 11, 1491

15. Brenner, I. B.; Zander, A.; Plantz, M.; Zhu, J. J. J. Anal. At. Spectrom. 1997, 12, 273.

16. Sung, Y.; Lim, H. B. J. Anal. At. Spectrom. 2001, 16, 767.

17. Pak, Y. N.; Koirtyohann, S. R. Bull. Korean. Chem. Soc. 1997, 18, 1167.

18. Alves, L. C.; Wiederin, D. R.; Houk, R. S. Anal. Chem. 1992, 64, 1155.

19. Alves, L. C.; Allen, L. A.; Houk, R. S. Anal. Chem. 1993, 65, 2468.

20. Minnich, M. G.; Houk, R. S. J. Anal. At. Spectrom. 1998, 13, 167.

21. Wiederin, D. R.; Houk, R. S.; Winge, R. K.; D'Silva, K. N. Anal. Chem. 1990, 62, 1155.

22. Alves, L. C.; Minnich, M. G.; Wiedrin, D. R.; Houk, R. S. J. Anal. At. Spectrom. 1994, 9, 399.

23. Gustavsson, A. Spectrochim. Acta 1988, 43B, 917.

24. Boumans, P. W. J. M.; Vrakking, J. J. A. M. Spectrochim. Acta 1985, $40 B, 1423$.

25. Boumans, P. W. J. M.; Vrakking, J. J. A. M. Spectrochim. Acta 1985, $40 B, 1107$.

26. Crain, J. S.; Alvarado, J. J. Anal. At. Spectrom. 1994, 9, 1223.

27. Minnich, M. G.; Houk, R. S.; Woodin, M. A.; Christiani, D. C. J. Anal. At. Spectrom. 1997, 12, 1345.

28. Pin, C.; Telouk, P.; Imbert, J. L. J. Anal. At. Spectrom. 1995, 10, 93.

29. Winge, R. K.; Fassel, V. A.; Peterson, V. J.; Floyd, M. A. Appl. Spectrosc. 1982, 36, 218.

30. Jakubowski, N.; Feldmann, I.; Stuewer, D. Spectrochim. Acta 1992, 47B, 107.

31. Garbarino, J. R.; Talor, H. E. Appl. Spectrosc. 1979, 33, 220

32. Saiwan, C. Ph.D. Thesis, Univ. of MO: 1991. 